\title{
Méthodes d'élaboration de cristaux massifs pour l'optique.
}

\author{
Bernard Ferrand \\ LETI/DOPT/SCOPI/LCDO/Matériaux/CEA-Grenoble \\ 17 rue des Martyrs, F-38054 GRENOBLE CEDEX 9, FRANCE \\ bernard.ferrand@cea.fr \\ http://www-leti.cea.fr
}

\begin{abstract}
Résumé. Depuis le début des années 80 , on assiste à un développement considérable des recherches sur les cristaux pour l'optique. L'avènement des diodes laser de puissance d'une part et l'exploitation des effets optiques non-linéaires dans les dispositifs optiques d'autre part ont contribué au développement de nouvelles sources lasers solides compactes et performantes donnant des émissions dans toute une gamme de longueurs d'onde ouvrant ainsi le champ à de nouvelles applications. Ces recherches ont nécessité l'élaboration de nouveaux cristaux laser et non-linéaires mais également relancé les études autour de cristaux connus en s'appuyant sur de nouveaux dopages par des terres rares ou des ions de transition. Toutes les techniques de croissance classiques ont été mises à contribution pour élaborer ces cristaux. Dans cet article nous décrirons ces principales techniques de croissance cristalline en s'attachant à développer des exemples concernant des cristaux ioniques ayant des applications en optique.
\end{abstract}

Nous décrirons plus particulièrement les méthodes de croissance faisant appel à une transformation liquide-solide. Parmi les techniques de croissance, on peut distinguer les méthodes à croissance rapide et les méthodes à croissance lente. Les méthodes à croissance rapide (quelques $\mathrm{mm} / \mathrm{h}$ ) opèrent aux températures élevées permettant d'obtenir de gros monocristaux et sont généralement caractérisées par une croissance dirigée à partir d'un bain fondu. Les méthodes à croissance lente (quelques $\mathrm{mm} / \mathrm{jour}$ ) procèdent généralement à des températures plus basses et s'imposent quand la fusion du cristal n'est pas congruente. Ce sont généralement les méthodes de croissance en solution faisant appel à un solvant et la synthèse hydrothermale. En fin de chapitre nous mentionnerons rapidement les techniques de transformation vapeur-solide permettant la croissance de monocristaux massifs.

Mots-clés. Monocristaux, cristallogenèse, matériaux pour l'optique.

\section{INTRODUCTION}

Le fondateur de la cristallogenèse scientifique est le professeur A. Verneuil. Dans un compte-rendu à l'Académie des Sciences en 1904, il décrit une technique de croissance par fusion à la flamme qui lui permet d'obtenir les premiers monocristaux de saphir et rubis [1]. En 1916 Jan Czochralski réalise la première expérience de tirage d'un fil d'étain monocristallin à partir d'un bain fondu $[\underline{2}, \underline{3}]$. Puis de nouvelles techniques de croissance apparaissent : Bridgman (1923), Kyropoulos (1926), Stockbarger (1936)... En réalité, dès la fin du XIX ${ }^{\mathfrak{e}}$ siècle, de nombreux cristaux avaient été synthétisés par des techniques à base de réactions chimiques et de solvants [4]. Plus que la pierre naturelle, le cristal synthétique va entrer dans la physique moderne comme la forme parfaite de la matière solide [ $[$ ].

Toutes ces techniques de cristallisation, pour se développer, vont devoir attendre soit des débouchés industriels, soit une meilleure compréhension de la physique du cristal, et surtout à partir des années 30, une meilleure compréhension des conceptions atomistiques de la cristallogenèse. 
L'intérêt croissant des cristaux pour la recherche depuis les années 50 va faire apparaître de nouveaux procédés d'élaboration et la cristallogenèse va permettre d'obtenir des cristaux que la nature ellemême n'a pas su créer. Les nombreux travaux qui lui sont alors consacrés vont déboucher sur d'extraordinaires applications techniques, notamment dans le domaine de l'électronique et de l'optique. L'importance des monocristaux demeure aujourd'hui essentielle pour les technologies présentes et futures []].

Les cristaux naturels se sont formés lentement par cristallisation de laves en refroidissement ou par évaporation lente. En laboratoire, quelques heures, voire quelques jours, suffisent pour faire croître un cristal. Pour obtenir un cristal il faut donner aux atomes la possibilité de se déplacer et de se réarranger correctement. R.A. Laudise a proposé une classification des méthodes de croissance qui résume la plupart des procédés actuels [7]. Parmi les techniques de croissance, on peut distinguer les méthodes à croissance rapide et les méthodes à croissance lente. Les méthodes à croissance rapide (quelques $\mathrm{mm} / \mathrm{h}$ ) opèrent aux températures élevées permettant d'obtenir de gros monocristaux et sont généralement caractérisées par une croissance dirigée à partir d'un bain fondu. Les méthodes à croissance lente (quelques $\mathrm{mm} / \mathrm{jour}$ ) procèdent à des températures plus basses et s'imposent quand la fusion du cristal n'est pas congruente. Ce sont généralement les méthodes de croissance en solution faisant appel à un solvant et la synthèse hydrothermale.

Si dès 1910, l'industrialisation de cristaux de rubis pour la joaillerie démarrait dans le laboratoire d'A. Verneuil à Paris (30 fours), puis dans l'usine Djevahidjian à Monthey en Suisse, c'est le développement de l'électronique et des dispositifs à semi-conducteurs qui va faire évoluer les techniques de cristallogenèse. La découverte du transistor dans les laboratoires Bell aux USA en 1950 va permettre de lancer le tirage d'un premier cristal industriel de grandes dimensions : le germanium [8]. Puis ce sera le silicium, ainsi que les semi-conducteurs III-V (AsGa, InP) et II-VI (CdTe).

La découverte en 1960 du premier cristal laser, le rubis ou alumine dopée chrome : $\mathrm{Al}_{2} \mathrm{O}_{3}: \mathrm{Cr}^{3+}$, obtenu par croissance Verneuil, lance le développement de la cristallogenèse des cristaux pour l'optique [9]. C'est d'ailleurs grâce au rubis que fut mis en évidence expérimentalement pour la première fois l'effet LASER. L'année suivante, l'effet laser est obtenu à $1,064 \mu \mathrm{m}$ dans un monocristal de $\mathrm{CaWO}_{4}$ dopé $\mathrm{Nd}$, obtenu par tirage Czochralski [10]. Puis ce fut la découverte du monocristal de grenat d'yttrium et aluminium : $\mathrm{Y}_{3} \mathrm{Al}_{5} \mathrm{O}_{12}$. Outre les cristaux lasers (à matrices ioniques ou à semi-conducteurs) [11], les applications faisant appel à des effets optiques non-linéaires ont permis le développement de nombreux nouveaux monocristaux [12].

\section{LES DIFFÉRENTES MÉTHODES D'ÉLABORATION DES CRISTAUX}

Dans cet article, nous décrirons les principales techniques de croissance cristalline en s'attachant à développer des exemples concernant des cristaux ioniques ayant des applications en optique. Comme évoqué précédemment, nous présenterons d'abord les techniques à croissance rapide utilisées pour les cristaux à fusion congruente, puis les techniques à croissance lente adaptées aux cristaux à fusion non congruente. Nous décrirons plus particulièrement les méthodes de croissance faisant appel à une transformation liquide-solide. En fin de chapitre nous mentionnerons rapidement les techniques de transformation vapeur-solide permettant la croissance de monocristaux massifs.

\subsection{Les méthodes à croissance rapide}

\subsubsection{La méthode Verneuil}

Le procédé de fusion à la flamme est resté pratiquement inchangé jusqu'à ce jour, comme l'avait décrit A. Verneuil au début du $20^{\mathrm{e}}$ siècle. Ses avantages sont liés à l'absence de creuset, à la possibilité d'obtenir de très hautes températures $\left(>2000^{\circ} \mathrm{C}\right)$ et à sa simplicité qui lui a conféré un exceptionnel développement industriel. La poudre du matériau à cristalliser est fondue dans la flamme d'un chalumeau oxhydrique. Son entraînement est réglé par le choc périodique d'un petit marteau et après passage dans le chalumeau, la poudre fondue vient cristalliser sur un germe. Celui-ci est placé sur un support mobile inférieur et protégé par un moufle réfractaire qui entoure le cristal naissant (ig. 1). 
La vitesse de croissance d'une boule de quelques dizaines de millimètres de diamètre est de l'ordre du $\mathrm{cm} / \mathrm{h}$, ce qui entraîne des contraintes importantes dans le cristal et une qualité cristalline pas toujours excellente. Ce sont surtout les forts gradients thermiques qui génèrent des contraintes et les inclusions de gaz qui expliquent la mauvaise qualité cristalline. Néanmoins dans l'industrie des lasers solides, les lasers à rubis et saphir-titane utilisent couramment des monocristaux d' $\mathrm{Al}_{2} \mathrm{O}_{3}$ dopé $\left(\mathrm{Cr}^{3+}\right.$ ou $\mathrm{Ti}^{3+}$ ), obtenus par méthode Verneuil. En France aujourd'hui la société RSA Le Rubis fabrique et commercialise des monocristaux de saphir obtenus par méthode Verneuil.

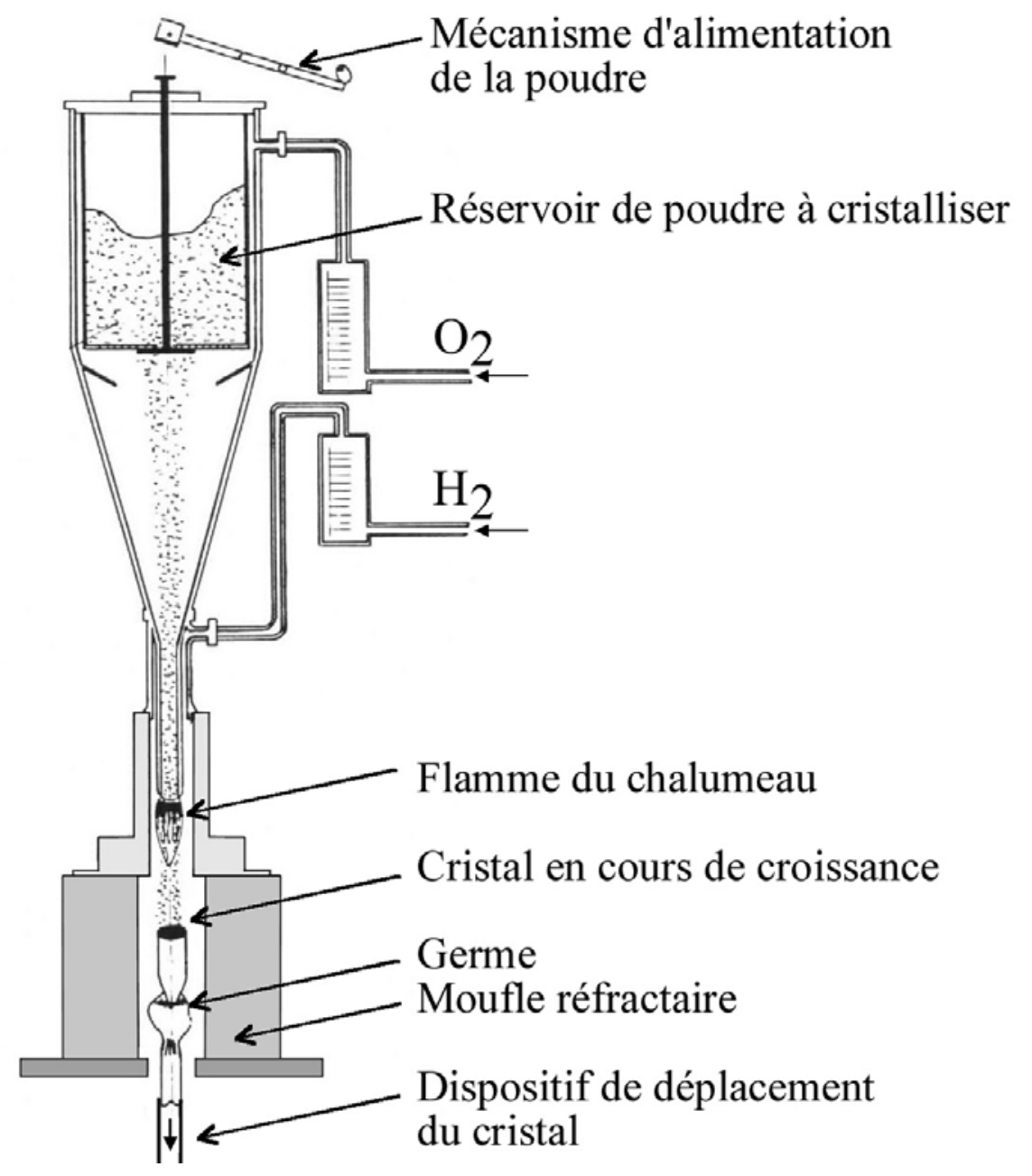

FIG. 1. Principe de la méthode Verneuil.

\subsubsection{La méthode Czochralski}

La technique la plus couramment utilisée aujourd'hui pour la production de nombreux monocristaux est le tirage Czochralski. Elle consiste à cristalliser le matériau à partir de sa phase liquide en trempant à la surface du bain fondu un germe monocristallin orienté du cristal à obtenir. Le bain se solidifie sur le germe légèrement plus froid. Le cristal formé est extrait en tirant lentement ce germe vers le haut. On peut contrôler le diamètre de croissance du cristal en faisant varier la température du bain ou la vitesse de tirage. Aujourd'hui on utilise une pesée du cristal (ou du creuset) pour contrôler le diamètre du cristal. La température du bain, du moins à l'interface, est fixée par l'équilibre solide-liquide. En réalité, on fait varier l'apport de puissance au bain, le cristal compensant la différence en générant plus ou moins de chaleur latente de solidification, donc en modifiant son diamètre. La pesée du cristal n'est pas utilisée pour le silicium car le solide est plus léger que le liquide. 


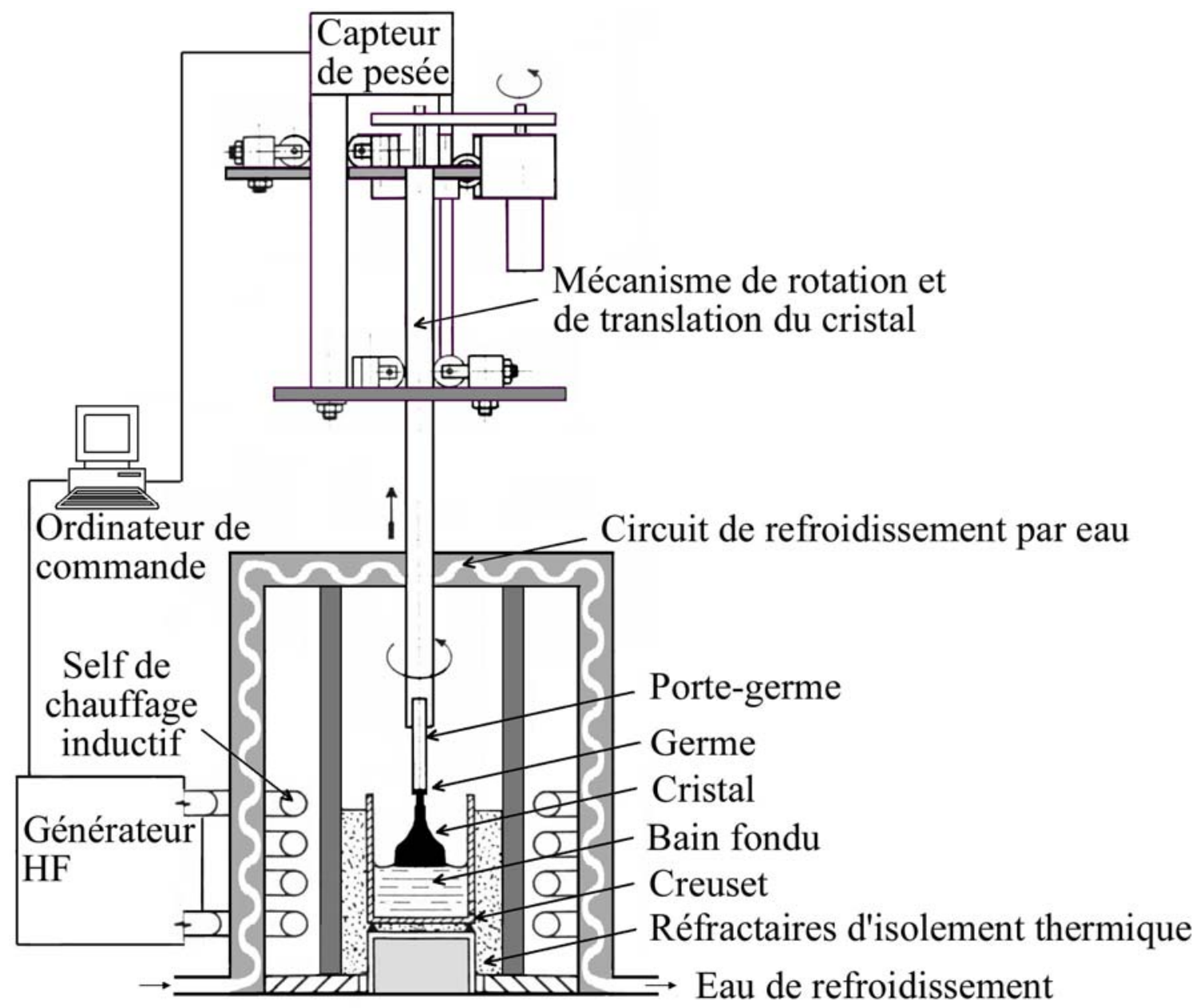

FIG. 2. Principe de la méthode de tirage Czochralski.

Un appareillage Czochralski est généralement composé de 2 parties : une partie supérieure supportant la tête de tirage et une partie inférieure où se trouve le creuset calorifugé, chauffé par induction haute fréquence (Fig. 2). Pour certains cristaux (silicium, $\mathrm{LiNbO}_{3}$, par exemple) un chauffage résistif peut être utilisé. L'ensemble doit pouvoir travailler aussi bien à l'air ambiant que sous gaz inerte ou sous vide. La perfection du cristal est souvent liée à la précision mécanique du dispositif de tirage, à la sensibilité de la pesée et au dispositif thermique entourant le creuset. L'interface de croissance que l'on peut ajuster en jouant sur la vitesse de rotation du germe doit être la plus plate possible afin d'éviter les tensions qui ont pour effet d'augmenter fortement la densité de dislocations. Du fait des hautes températures de fusion, les creusets doivent être constitués d'un matériau neutre chimiquement vis-à-vis du matériau à élaborer et de l'atmosphère, ce qui impose des métaux nobles : platine ou iridium pour les oxydes. En particulier l'utilisation de l'iridium interdit de travailler en atmosphère riche en oxygène. Dans le cas de semi-conducteurs ou de matériaux nonoxydes l'utilisation de creusets en quartz ou carbone peut être envisagée.

La croissance est généralement décomposée en 3 phases :

- l'affinage au diamètre du germe (quelques millimètres) qui permet d'éliminer les défauts en provenance de celui-ci ;

- la réalisation de la tête du cristal qui consiste à atteindre plus ou moins rapidement le diamètre nominal du cristal $(25,50,75,100 \ldots$ millimètres $)$, ce qui permet de diminuer certains défauts du type dislocations par exemple ;

- le tirage au diamètre qui doit être le plus stable possible, sans fluctuations de diamètre, sans à-coups de puissance et de translation et qui peut durer plusieurs dizaines d'heures. 
En fin de tirage le cristal est soulevé lentement du bain, puis une rampe de refroidissement est appliquée.

Les vitesses de croissance utilisées en technique Czochralski sont de l'ordre de quelques $\mathrm{mm} /$ heure pour les matériaux non dopés. En cas de dopage, notamment en vue d'un effet laser (néodyme dans YAG par exemple), il est nécessaire de diminuer fortement cette vitesse jusqu'à quelques dixièmes de $\mathrm{mm}$ par heure [13]. Cette réduction a pour effet d'éviter les défauts liés à des phénomènes locaux de surfusion (appelés striations) et d'obtenir une concentration en dopant la plus homogène possible dans le cristal. Ce problème est particulièrement critique dans le cas de substitutions de cations ayant des rayons ioniques très différents.

Pour certains cristaux particulièrement sensibles aux chocs thermiques $\left(\mathrm{LiNbO}_{3}\right.$ ou $\mathrm{LaTiO}_{3}$ par exemple), un four auxiliaire de recuit peut être ajouté [14]. Les conditions de croissance dépendent fortement des cristaux étudiés. Outre les grenats (aluminates, gallates...), niobates et tantalates de lithium, la méthode Czochralski permet d'obtenir de nombreux monocristaux (Fig. 3) : aluminates $\left(\mathrm{YAlO}_{3}, \mathrm{LaMgAl} 1_{11} \mathrm{O}_{19}\right)$, silicates $\left(\mathrm{Y}_{2} \mathrm{SiO}_{5}, \mathrm{Gd}_{2} \mathrm{SiO}_{5}, \mathrm{Lu}_{2} \mathrm{SiO}_{5}\right)$, germanates $\left(\mathrm{Bi}_{4} \mathrm{Ge}_{3} \mathrm{O}_{12}, \mathrm{Bi}_{12} \mathrm{GeO}_{20}\right)$, vanadates $\left(\mathrm{YVO}_{4}\right)$, borates $\left(\mathrm{YCa}_{4} \mathrm{~B}_{3} \mathrm{O}_{10}, \mathrm{GdCa}_{4} \mathrm{~B}_{3} \mathrm{O}_{10}\right)$, tungstates $\left(\mathrm{CaWO}_{4}\right)$, mais également fluorures $\left(\mathrm{LiYF}_{4}\right.$ notamment [타] $)$.

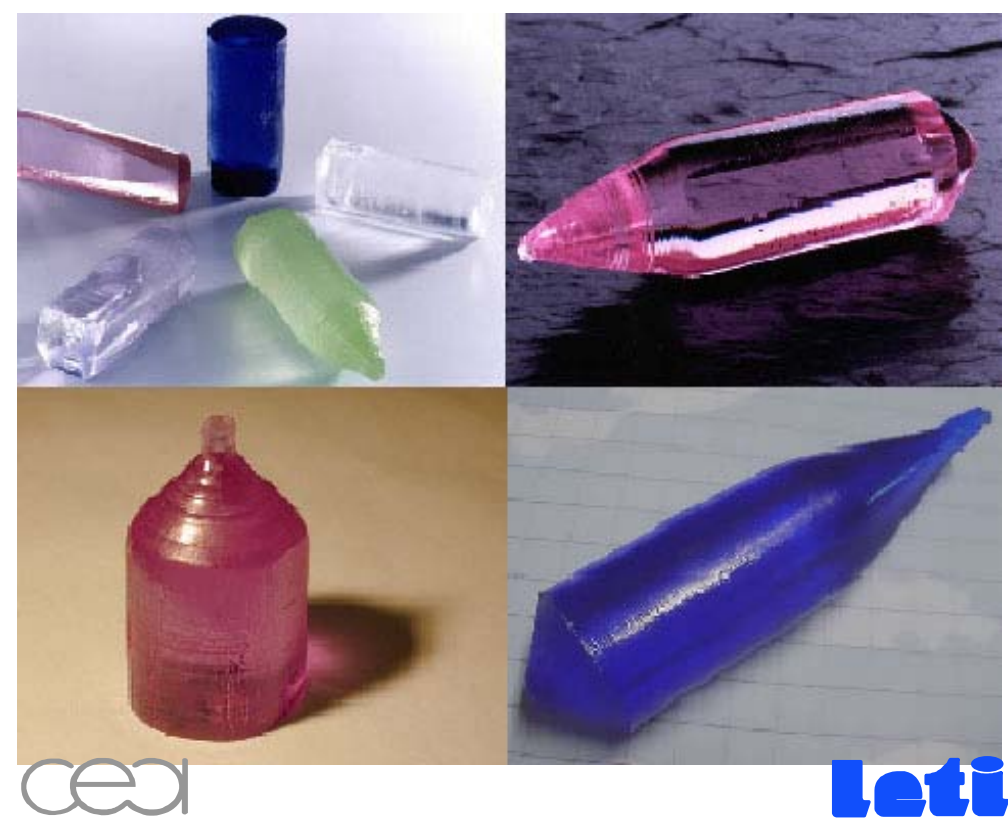

FIG. 3. Exemples de monocristaux obtenus par tirage Czochralski (CEA/LETI/DOPT). (a) $\mathrm{Y}_{2} \mathrm{SiO}_{5}$ non dopé et dopé $\mathrm{Er}, \mathrm{Yb}, \mathrm{Tm}$ et $\mathrm{Cr}$ : cristaux émetteurs lasers ; (b) $\mathrm{CaWO}_{4}$ dopé $\mathrm{Nd}$ : cristal laser autour de 1,06 $\mu \mathrm{m}$; (c) $\mathrm{Nd}: \mathrm{GdCa}_{4} \mathrm{~B}_{3} \mathrm{O}_{10}$ : cristal laser et doubleur de fréquences; (d) $\mathrm{LaMgAl}_{11} \mathrm{O}_{19}$ dopé Co : absorbant saturable à $1,55 \mu \mathrm{m}$.

Pour la croissance de certains matériaux qui doivent être obtenus en grand diamètre, une méthode Czochralski modifiée a été mise au point, c'est la méthode Kyropoulos. Le concept de base est le même que pour le Czochralski mais après démarrage de la croissance, la translation est stoppée et le contrôle de la croissance est réalisé par une diminution de la puissance du générateur à haute fréquence. Cette méthode est essentiellement utilisée pour la croissance de gros cristaux d'halogénures alcalins (NaI, CsI...).

Certains monocristaux semi-conducteurs et composés optoélectroniques (GaAs, InP...) sont difficiles à obtenir en raison de la dissociation du bain en cours de croissance. Une des solutions retenues pour éviter cet inconvénient consiste à emprisonner le bain [16]. Ceci a permis de mettre au point des techniques de tirage, soit à travers un encapsulant, soit dans une ampoule scellée, soit sous pression contrôlée (quelques atmosphères pour GaAs). 


\subsubsection{La méthode Bridgman-Stockbarger}

La méthode Bridgman-Stockbarger consiste à abaisser lentement un bain fondu dans un gradient de température faisant passer le bain de l'état liquide à l'état solide. À l'origine la méthode Bridgman consistait à réaliser une croissance sous tubes horizontaux (voir méthode fusion de zone). L'appellation Bridgman-Stockbarger a été donnée à la croissance en creusets verticaux qui peut être réalisée sous différentes configurations thermiques (Fig. 4). Cette méthode a été développée essentiellement pour la croissance des semi-conducteurs, mais a également trouvé de nombreuses applications pour les cristaux d'halogénures alcalins et de fluorures.

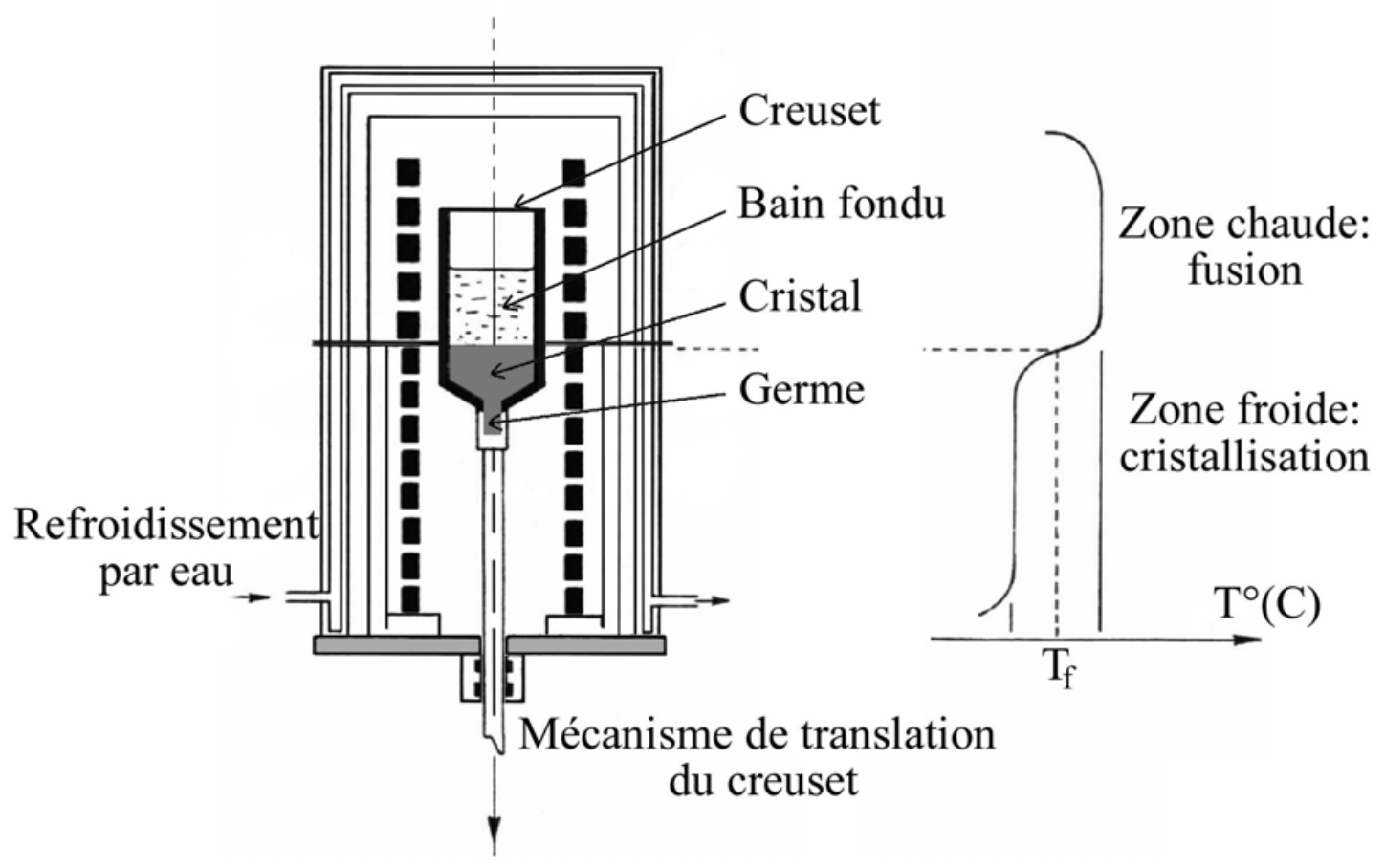

FIG. 4. Principe de la méthode de tirage Bridgman.

Le matériau à cristalliser est placé dans un creuset que l'on place dans la zone chaude du four à une température supérieure à la température de fusion $\left(\mathrm{T}_{\mathrm{f}}\right.$ ). Le creuset est alors translaté lentement au moyen d'une tige support dans une zone froide où le cristal se solidifie. Afin d'assurer un bon gradient de température et assurer une interface solide-liquide la plus homogène possible, un écran thermique peut être utilisé pour bien délimiter les deux zones. Pour ces matériaux l'utilisation d'un creuset en graphite est particulièrement adaptée et d'une réalisation aisée. Le creuset à base conique en pointe qui permet un bon contrôle du début de croissance en favorisant le démarrage d'un cristal unique. Un germe peut également être placé dans un appendice situé à la pointe du creuset. La croissance de matériaux du type oxyde se heurte à la problématique du creuset et en particulier au démoulage souvent difficile des cristaux. Le choix du mode de chauffage dépend des matériaux et des conditions de croissance nécessaires. Il existe aussi bien des fours résistifs (résistance en graphite par exemple) que des fours à chauffage inductif. Les vitesses de translation sont de l'ordre de quelques millimètres par heure. Cette méthode est particulièrement adaptée à une cristallisation industrielle : gros cristaux, cristaux en forme ou plusieurs cristaux simultanément (Fig. 5).

Parmi les cristaux pour l'optique, outre les fluorures $\left(\mathrm{LiYF}_{4}, \mathrm{MgF}_{2}, \mathrm{CaF}_{2}, \mathrm{BaF}_{2} \ldots\right)$, quelques monocristaux d'oxydes peuvent également être obtenus par méthode Bridgman $\left(\mathrm{YAlO}_{3}, \mathrm{Bi}_{4} \mathrm{Ge}_{3} \mathrm{O}_{12}\right.$, $\left.\mathrm{Y}_{2} \mathrm{O}_{3} \ldots\right)$. Suivant la nature du matériau et du creuset, il est possible d'obtenir par méthode Bridgman, des monocristaux préformés de forme simple - cylindres, parallélépipèdes... - donnés par la forme du creuset. 

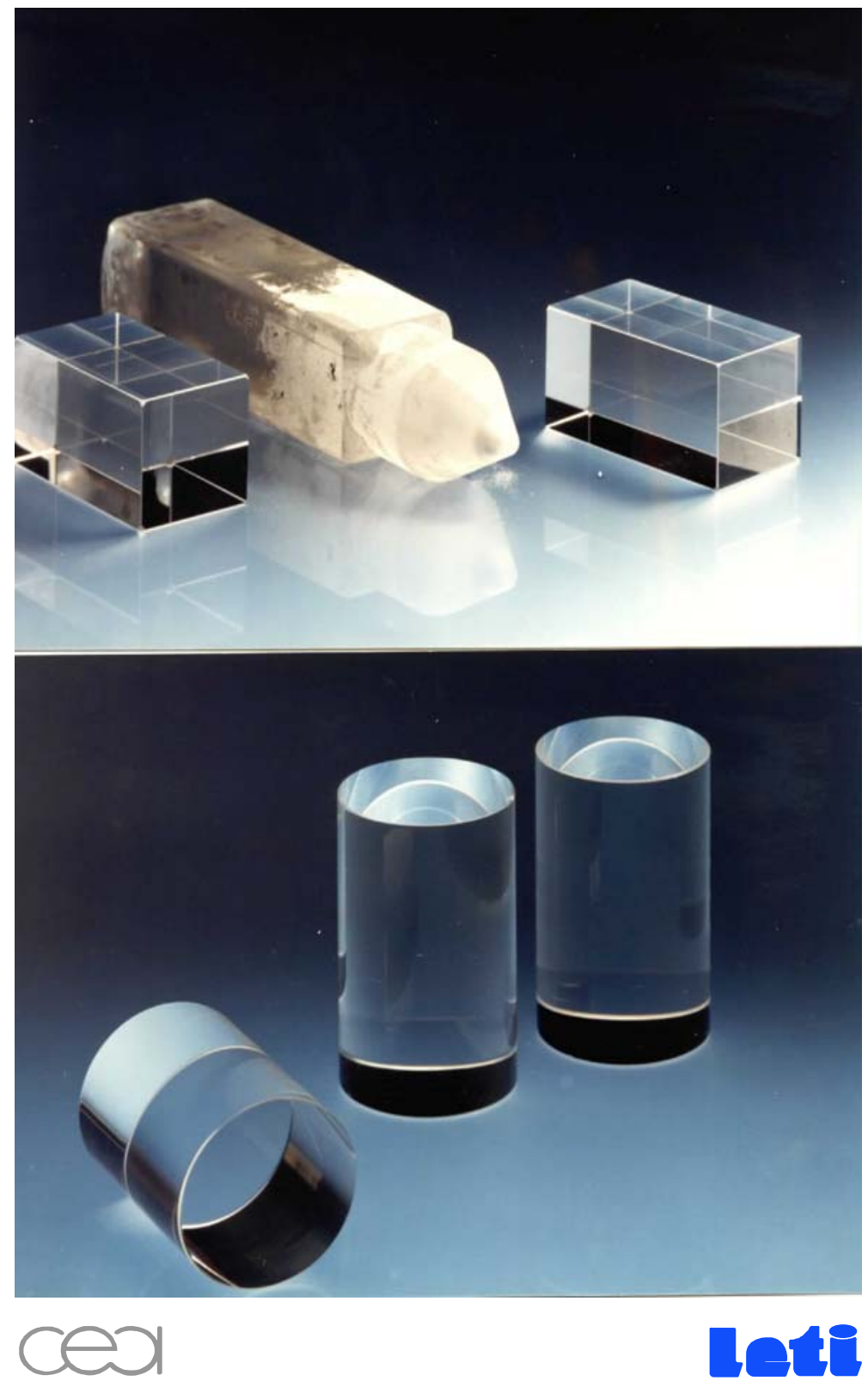

FIG. 5. Monocristaux de $\mathrm{BaF}_{2}$ obtenus par méthode Bridgman.

De nombreuses modifications ont été apportées à cette technique en cherchant notamment à conserver le creuset fixe. Ainsi s'est développée la méthode de l'échangeur thermique (HEM) qui associe la présence d'un germe, placé au fond du creuset, avec un échangeur de chaleur qui permet d'extraire à la partie inférieure du lingot, la chaleur latente de solidification (Fig. 6). Les premiers travaux ont porté sur le saphir pur et dopé et ont abouti à la production de monocristaux de $70 \mathrm{~mm}$ de diamètre et de $10 \mathrm{~mm}$ d'épaisseur, en vue d'applications telles que les lasers ou l'électro-optique [17]. D'autres matrices monocristallines ont alors été envisagées à des fins commerciales, telles que $\mathrm{Y}_{3} \mathrm{Al}_{5} \mathrm{O}_{12}$ dopé au néodyme (YAG:Nd) et $\mathrm{MgF}_{2}$ dopé au cobalt pour les matériaux lasers. Certains matériaux se sont développés industriellement et présentent une meilleure qualité cristalline lorsqu'ils sont produits par $\mathrm{HEM}\left(\mathrm{Al}_{2} \mathrm{O}_{3}, \mathrm{Bi}_{4} \mathrm{Ge}_{3} \mathrm{O}_{12} \ldots\right)$, la raison se trouvant, avant tout, dans la forme (convexe) et dans la position (par rapport au liquide) particulières de l'interface durant la croissance, l'absence de mouvement évitant de la perturber [18]. 


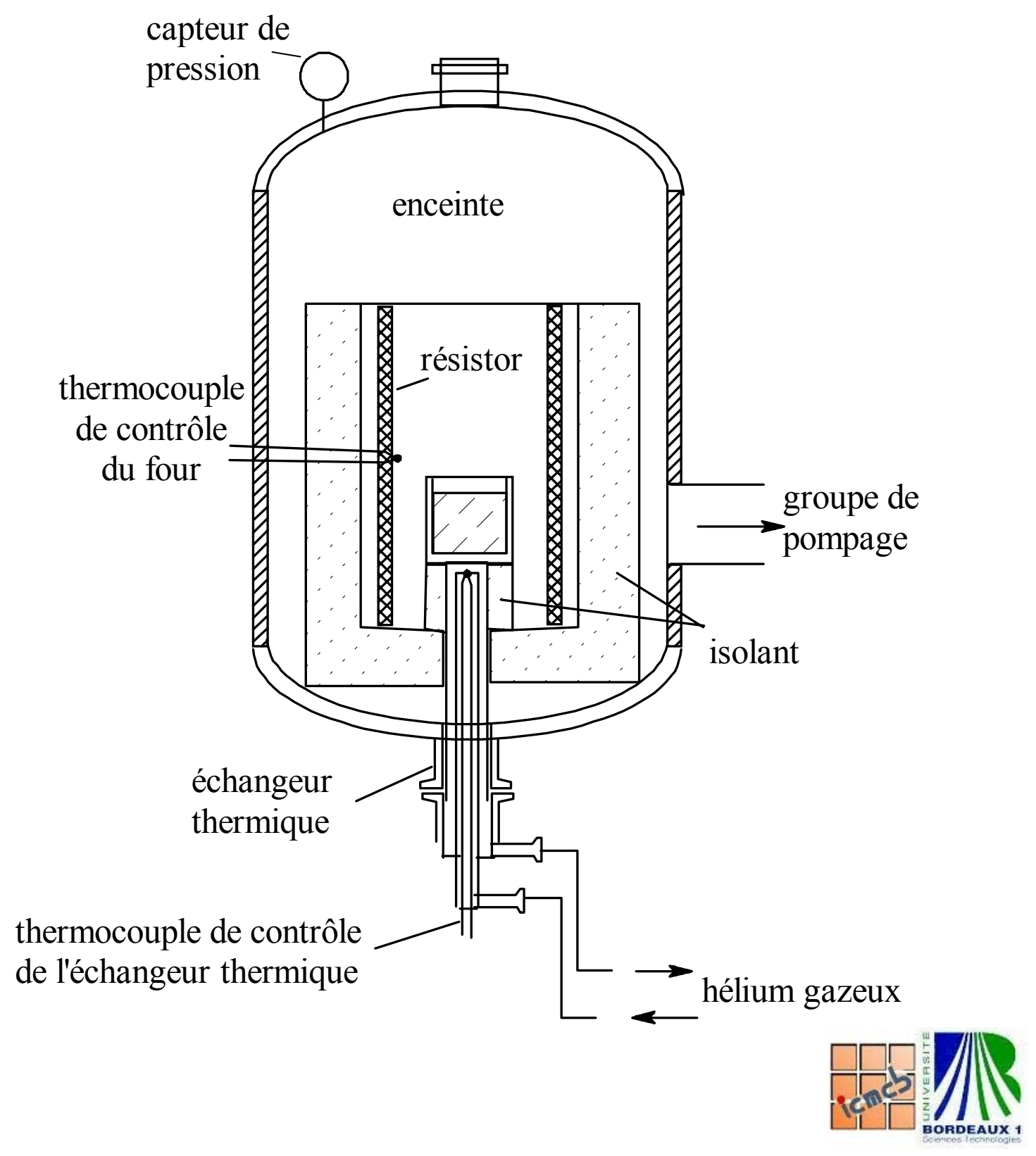

FIG. 6. Principe de la méthode HEM [18].

\subsubsection{La méthode de fusion de zone}

Dérivée de la méthode Bridgman, la fusion de zone (verticale ou horizontale) est surtout considérée comme une méthode de purification des matériaux. Elle peut cependant être utilisée pour la croissance de monocristaux. Dans ce cas le matériau est préparé sous forme d'un barreau polycristallin, monté généralement verticalement sur un support, dont une partie va être fondue localement. La préparation du barreau polycristallin est un point commun crucial entre toutes ces techniques. Le bain fondu est suspendu comme une goutte entre les 2 parties du barreau. Cette zone fondue est ensuite déplacée lentement, traverse le barreau et par refroidissement un monocristal peut être généré à partir de la zone non-fondue du barreau ou sur un germe amené au contact du barreau si une de ses extrémités a été fondue (Fig. 7). On parle alors de méthode de la zone flottante puisque la zone fondue créée dans le barreau est supportée par les forces de tension superficielle du liquide. Il n'y a donc pas de creuset comme source de contamination et cette méthode, délicate à mettre en oeuvre, fournit des cristaux de haute pureté. La fusion du matériau peut être obtenue par différentes techniques : induction haute fréquence, concentration d'un rayonnement lumineux ou effet Joule à l'aide d'une résistance extérieure 
ou partiellement immergée dans la zone liquide. L'utilisation d'une résistance (lame de platine percée de trous par exemple), amenée au contact du barreau permet une fusion de celui-ci. Si un germe est amené au contact de l'autre face de la lame puis déplacé vers le bas, la croissance peut démarrer et cette méthode (appelée résistor plan) peut permettre la croissance de cristaux de grandes dimensions. La croissance sous atmosphère contrôlée est également possible.

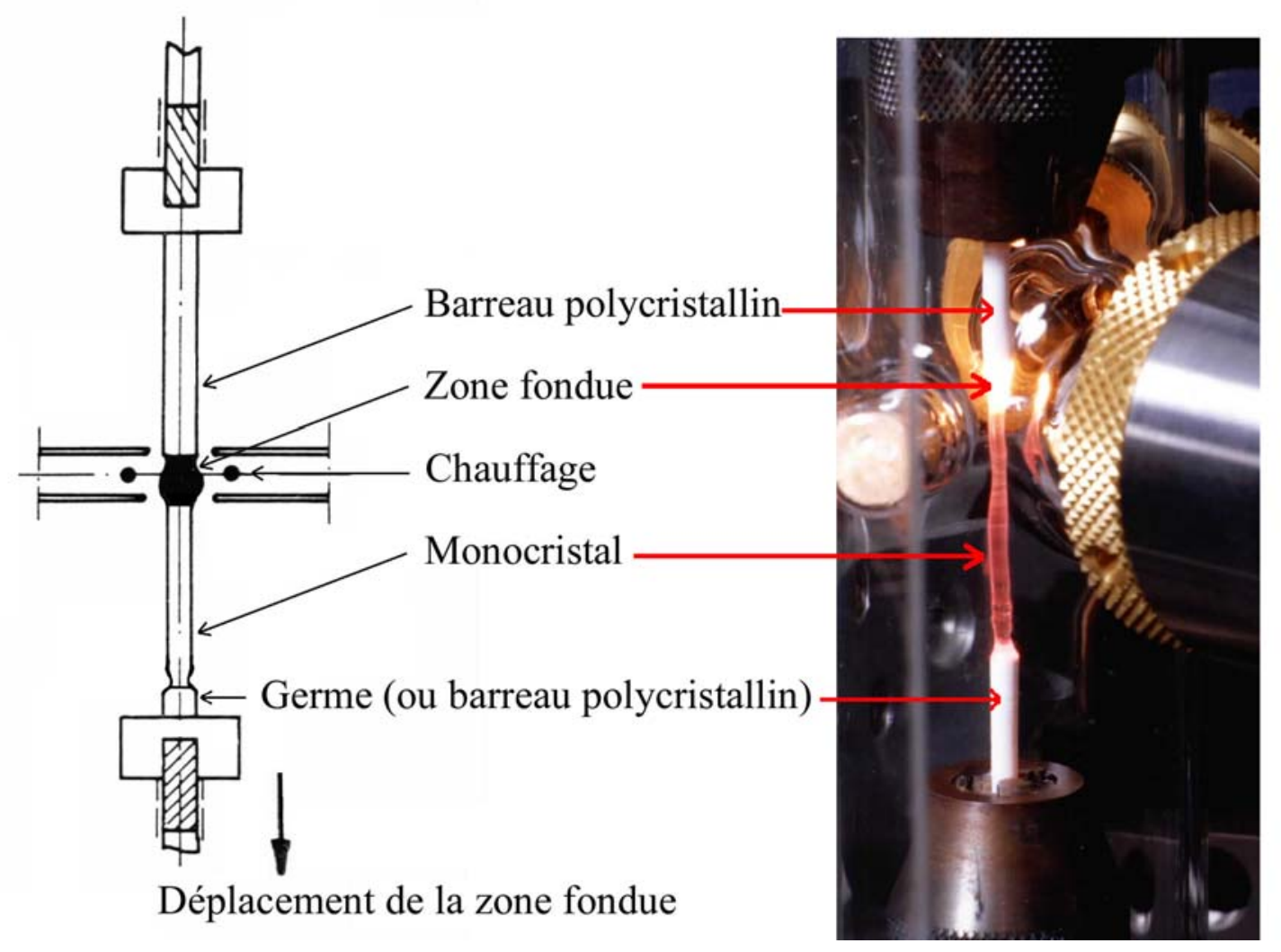

FIG. 7. Principe de la méthode de Fusion de Zone. Exemple d'un chauffage par laser CO2 (exemple d'un cristal de $\mathrm{Nd}: \mathrm{YAG})$.

Ce sont les modes de chauffage par concentration d'un rayonnement lumineux qui se sont le plus développés ces dernières années : lampes (méthode du four à image) ou laser $\mathrm{CO}_{2}$. Cette dernière méthode appelée LHPG, "Laser Heated Pedestal Growth» consiste à provoquer la fusion par utilisation du rayonnement d'un laser $\mathrm{CO}_{2}$ continu focalisé sur le barreau fritté [19]. Une possibilité intéressante de ces méthodes est le tirage de fibres monocristallines de petits diamètres $(500 \mu \mathrm{m}$ à $1 \mathrm{~mm}$ ) qui peuvent avoir un certain intérêt en recherche et dans certaines applications [20]. $\mathrm{LiNbO}_{3}$ est un des matériaux optiques les plus étudiés par ces différentes méthodes de fusion de zone.

\subsubsection{La croissance de cristaux préformés}

Pour obtenir des cristaux préformés par technique de tirage, il faut mettre en jeu une technique de filière. Le préformage dérive directement de la méthode de croissance Czochralski. Stepanov est le premier qui pallie l'instabilité de la méthode Czochralski en utilisant une filière flottant sur le bain (wetting die) qui fixe les dimensions du cristal et rend évanescentes les perturbations au cours du tirage [1ㅡ]. Le préformage est né, connu sous l'appellation Edge-defined Film fed Growth (EFG) et les développements ultérieurs conduiront à l'alimentation capillaire pour l'élaboration de sections plus fines [22]. Le préformage permet l'élaboration de formes creuses, minces et très allongées dans des matériaux durs et fragiles, comme cela a été étudié dans le saphir. De telles propriétés mécaniques interdisent tout autre procédé tel que l'extrusion ou l'usinage. Enfin, pour certaines pièces la croissance capillaire vient directement concurrencer les techniques de moulage sans l'inconvénient du contact avec le creuset, ni problème de dilatation différentielle. L'intérêt du préformage augmente 
d'autant plus que des tolérances dimensionnelles sévères sont respectées. On réalise couramment en préformage une précision de $0,1 \mathrm{~mm}$ (Fig. 8) .

En préformage classique, le ménisque régit localement la dimension du solide préformé, tandis que sa géométrie globale dépend des compositions de mouvement de l'axe de tirage [23]. Cette technique de croissance permet l'élaboration de formes simples (tubes, plaques, barreaux). Des adaptations de la technique ont permis l'élaboration de formes plus complexes, tubes et dômes notamment (Fig. 9).

\section{Principe du préformage :}

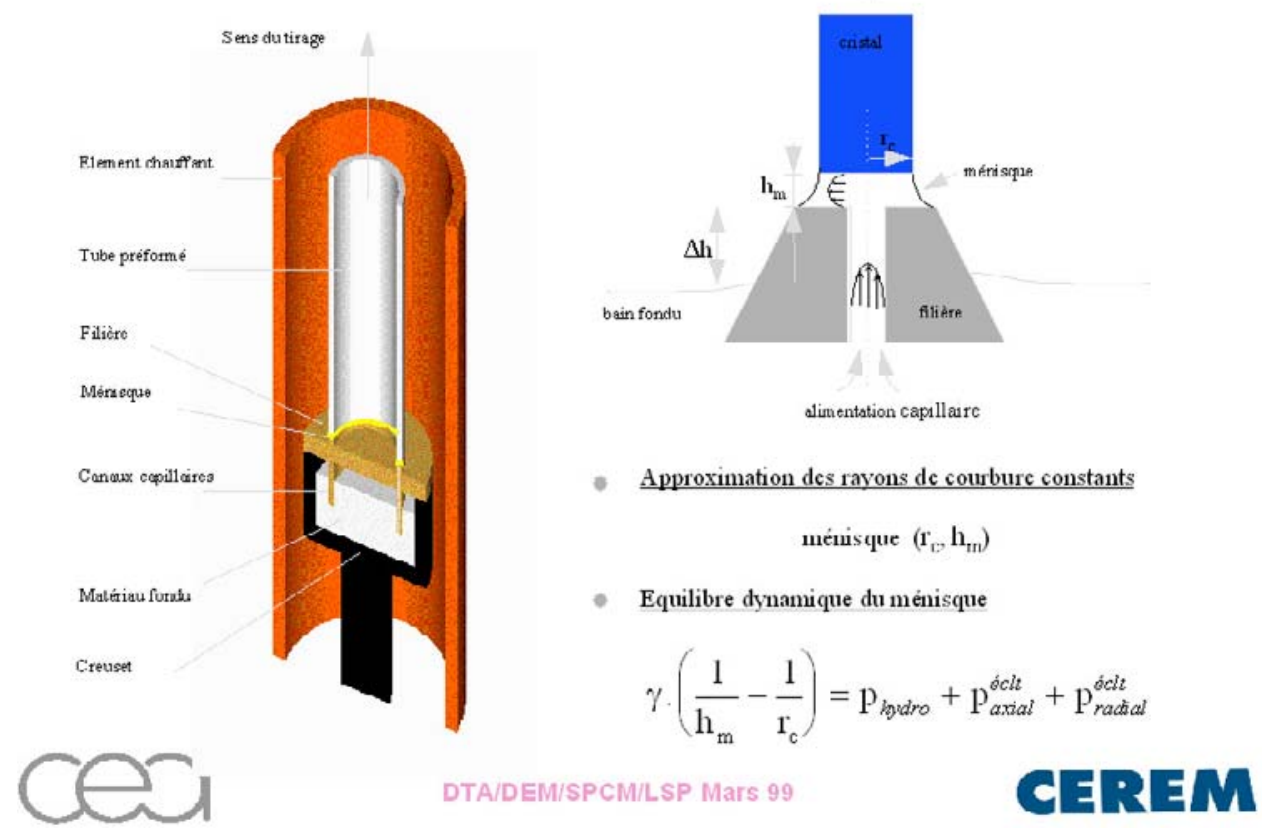

FIG. 8. Principe de la méthode EFG [23].

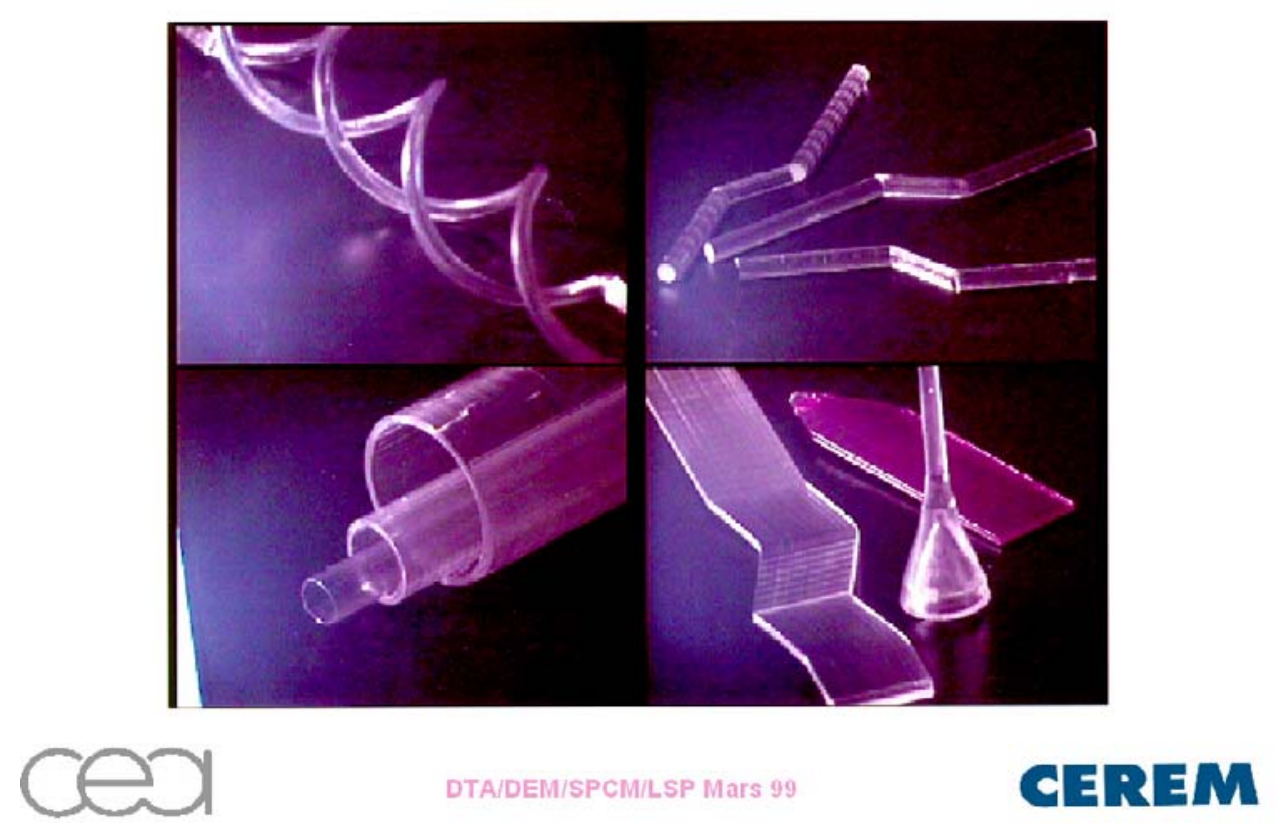

FIG. 9. Élaboration de monocristaux en forme de barreaux, de tubes et de plaques de saphir par méthode EFG. 


\subsection{Les méthodes à croissance lente}

Lorsqu'un matériau ne présente pas une fusion congruente ou bien possède une transition de phase à haute température, il faut utiliser une méthode de croissance en solution qui nécessite l'emploi d'un solvant. Dans ce cas la croissance a lieu à partir d'une solution sursaturée, à plus basse température avec des vitesses très faibles pouvant descendre à quelques millimètres par jour. Les cristaux obtenus sont généralement plus petits mais souvent de bonne qualité, exempts de tensions, mais pouvant être contaminés par le solvant. Les méthodes sont les méthodes de croissance en solution, en solvant aqueux, organique ou inorganique, sels fondus et la croissance hydrothermale.

\subsubsection{La croissance en solution aqueuse}

La cristallisation en solution aqueuse est utilisée pour tous les cristaux solubles dans l'eau. La sursaturation de la solution peut être obtenue, soit par abaissement graduel de la température, soit par évaporation progressive et contrôlée de l'eau (Fig. 10). Dans le premier cas il s'agit d'assurer très précisément la décroissance en température $\left(0,01\right.$ à $0,1{ }^{\circ} \mathrm{C} /$ jour $)$ pendant plusieurs mois. Les cristaux formés peuvent présenter des tensions dues aux variations de température. Dans le second cas, la croissance se fait à température constante, mais il est difficile de contrôler l'évaporation constante pour maintenir avec une précision suffisante la vitesse de cristallisation.

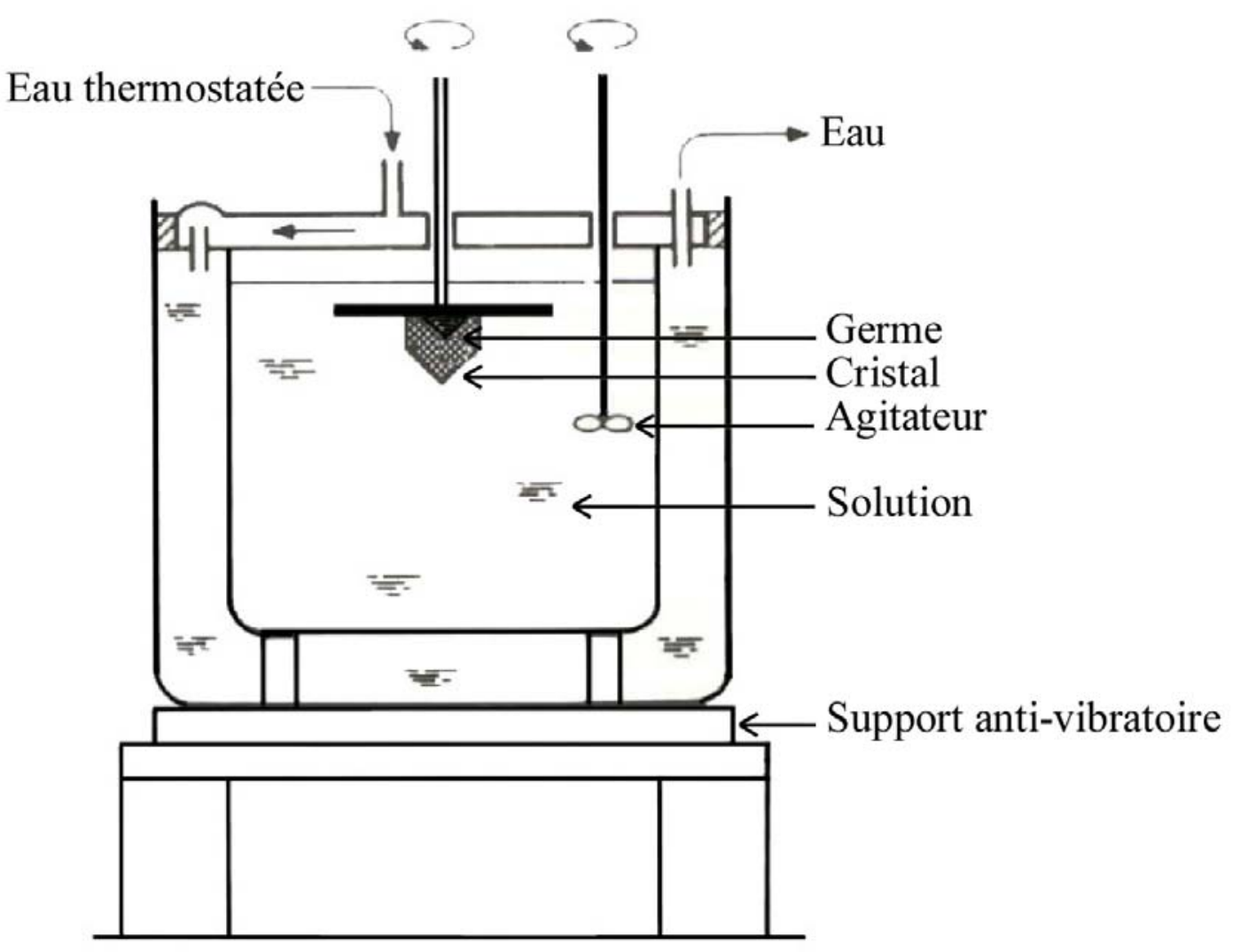

FIG. 10. Principe de la méthode de croissance en solution aqueuse.

La croissance en solution aqueuse est utilisée pour la production d'halogénures alcalins, $\mathrm{HIO}_{3}$, et des cristaux de la famille du $\mathrm{KDP}\left(\mathrm{KH}_{2} \mathrm{PO}_{4}\right.$ ou dihydrogénophosphate de potassium). Ce cristal utilisé dans le projet Mégajoule a fait l'objet de nombreuses études afin d'augmenter la vitesse de croissance. En effet l'utilisation nécessite de gros cristaux et un nouveau procédé de croissance a été mis au point au Laboratoire Lawrence Livermore de Californie. Des pyramides de KDP de plus de 300 kilogrammes ont été obtenues en 52 jours (Fig. 11). Pour créer de telles pièces $(66 \times 53 \times 58 \mathrm{~cm})$, les chercheurs américains ont utilisé une technique dite de croissance rapide mise au point pour la 
première fois en Russie et actuellement développée en France par Saint-Gobain Cristaux et Détecteurs en collaboration avec le $\underline{\text { CEA [24] }}$.

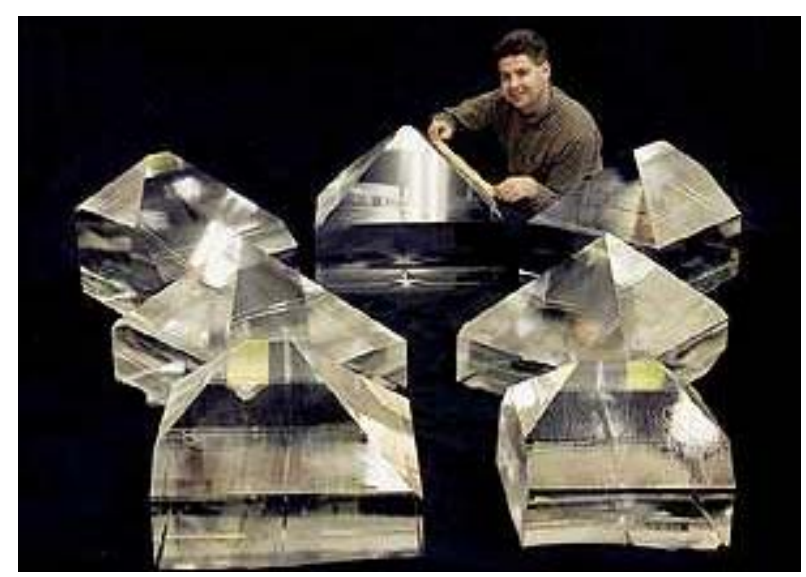

FIG. 11. Monocristaux de KDP obtenus par croissance en solution au Lawrence Livermore National Laboratoire. Informations disponibles sur le site $:$ http://www.llnl.gov/nif/nif.html.

\subsubsection{La croissance en solvant (flux)}

Les méthodes de croissance en flux sont voisines des méthodes de croissance en solution aqueuse. Le matériau à cristalliser (soluté) est mélangé à un sel (solvant) et le mélange est porté à haute température jusqu'à le rendre liquide. Le solvant est choisi de telle sorte que la température de fusion du mélange soit inférieure à celle du soluté. C'est un des avantages de la méthode, surtout pour les matériaux fondant à très haute température. La difficulté principale de cette méthode est le choix du solvant. Celui-ci doit dissoudre une quantité importante de soluté sans réagir avec lui et de plus il ne doit pas réagir avec le creuset dans lequel a lieu la cristallisation. Les flux les plus couramment utilisés sont : l'oxyde de plomb, de baryum, de bore, de bismuth ou le fluorure de plomb, de calcium ou de sodium [4].

Plusieurs cristaux utilisés en optique non-linéaire $-\mathrm{KTiOPO}_{4}(\mathrm{KTP}), \mathrm{BaB}_{2} \mathrm{O}_{4}(\mathrm{BBO}), \mathrm{LiB}_{3} \mathrm{O}_{5}$ (LBO)...- sont obtenus par la méthode de flux. En France la société Cristal Laser commercialise les cristaux de la famille du KTP ainsi que le LBO. La technique de base, consistant à enfermer l'ensemble soluté-solvant dans un creuset fermé puis à programmer un refroidissement lent, ne permet pas un contrôle continu de la croissance (Fig. 12). La création d'un point froid pour provoquer le démarrage de la croissance et diminuer le nombre de cristaux, tout en augmentant leur taille, n'a pas conduit à un procédé industriel (travaux de $\mathrm{W}$. Tolskdorf sur $\mathrm{Y}_{3} \mathrm{Fe}_{5} \mathrm{O}_{12}[\underline{25}]$ ).

Afin de pouvoir disposer d'un suivi constant et visuel de la croissance, s'est développée une technique dérivée de la méthode Czochralski, le tirage à partir du flux ou Top-Seeded-SolutionGrowth (TSSG). Le bain fondu est constitué d'un mélange soluté-solvant et la croissance est provoquée par la trempe d'un germe à la surface du bain dont la température doit être ajustée très précisément [26]. En effet il s'agit de se trouver à la température de sursaturation du bain afin d'éviter, soit une dissolution du germe, soit une nucléation spontanée importante. La croissance démarre ensuite par une translation très lente du germe (de l'ordre du millimètre par jour) et un contrôle très précis du refroidissement $\left(0,1^{\circ} \mathrm{C}\right.$ par jour). Comme dans la méthode Czochralski, un contrôle de la croissance peut être réalisé par la pesée en continu du cristal. 


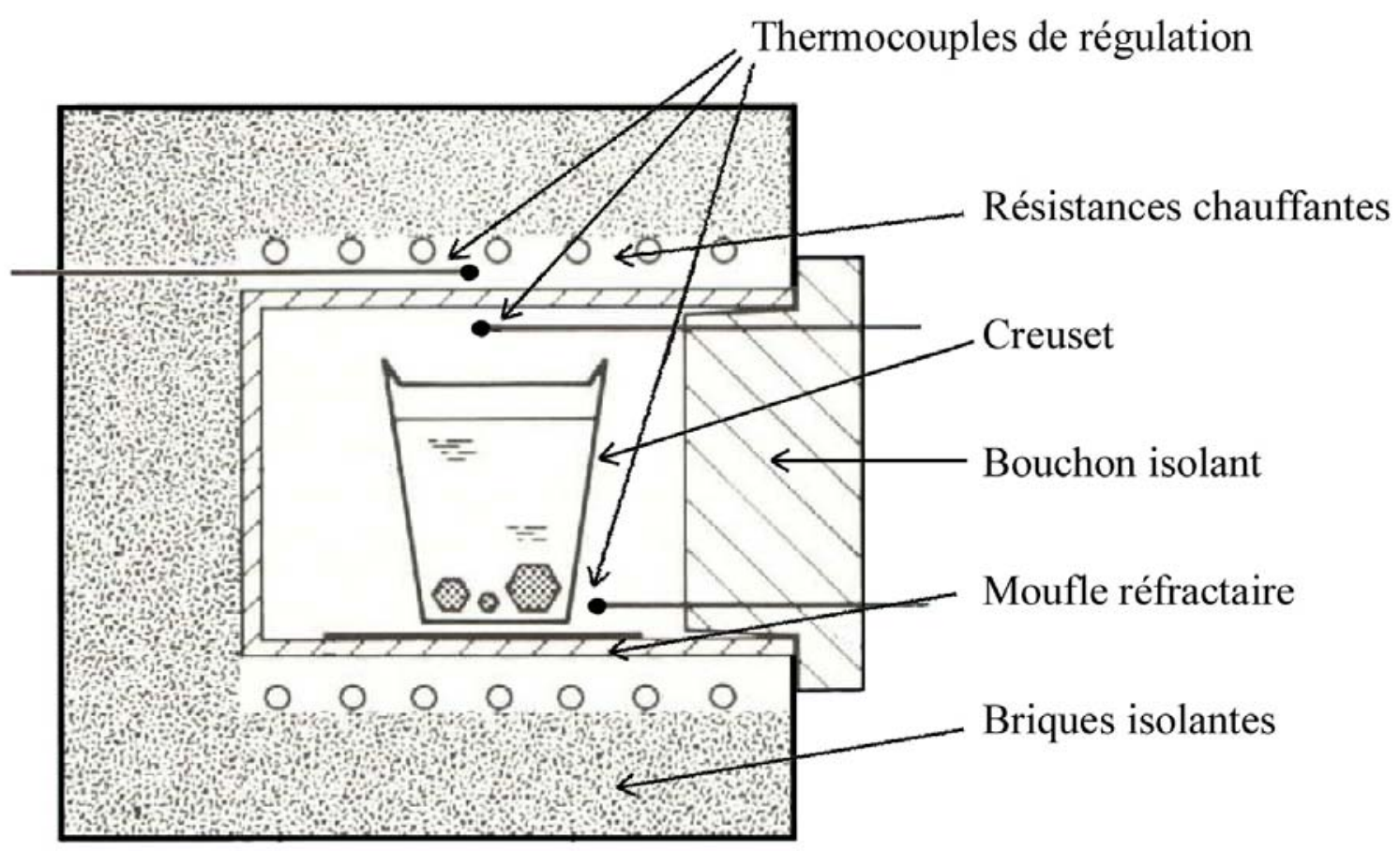

FIG. 12. Principe de la méthode de croissance dans le flux.

\subsubsection{La croissance hydrothermale}

Le terme hydrothermal est utilisé pour décrire l'ensemble des méthodes dans lesquelles la cristallisation de matériaux relativement insolubles dans les conditions ordinaires est obtenue à partir d'une solution aqueuse chauffée sous haute pression. Le matériau à recristalliser (ou corps mère) et une quantité mesurée de solution sont placés dans un autoclave cylindrique en acier fermé hermétiquement. L'ensemble est porté à une température telle que la pression soit suffisante. La combinaison des deux paramètres, température et pression, entraîne une dissolution importante du corps mère. Si la solution devient sursaturée au point de l'autoclave où se trouve un germe, le matériau en excès cristallise sur le germe [27].

Un des procédés les plus utilisés pour obtenir cette sursaturation est l'établissement d'un gradient de température entre la zone où se trouve le corps mère (zone basse) et celle où se trouve le germe (zone haute). Généralement le corps mère est placé dans la partie chaude de l'autoclave et le germe dans la partie froide. Un écran convenablement percé (5 à $20 \%$ d'ouverture) sépare les deux zones de dissolution et de cristallisation et les rend isothermes. Dans la zone de dissolution, la solution se sature en corps mère, les courants de convection créent alors un mécanisme de transport, par différence de densité, jusqu'à la zone de croissance plus froide où la solution devient sursaturée. Le matériau corps mère cristallise alors sur le germe. L'amplitude de la sursaturation est déterminée par la différence de température entre le bas et la haut de l'autoclave. Un cycle s'établit: dissolution, transport, croissance... et il y a transport continu du corps mère de la zone de dissolution vers la zone de cristallisation ( $\underline{\text { Fig. 13 }}$ ).

La méthode hydrothermale est donc une technique relativement simple, mais sa mise en oeuvre nécessite un appareillage très spécialisé (autoclaves en aciers spéciaux, réseau haute pression pouvant atteindre plusieurs milliers de bars). Les avantages sont liés aux relativement basses températures $\left(500{ }^{\circ} \mathrm{C}\right)$ ce qui permet la croissance de cristaux de bonne qualité avec des vitesses de croissance de l'ordre du mm/jour. Les grenats, $\mathrm{TiO}_{2}$, mais surtout le quartz et aujourd'hui $\mathrm{AlPO}_{4}$, sont les principaux cristaux obtenus par cette méthode. La société SICN à Annecy développe et maîtrise la fabrication de quartz de synthèse par la méthode de recristallisation hydrothermale. $\mathrm{KTiOPO}_{4}$ est également un cristal préparé par voie hydrothermale. Un certain nombre d'avantages apparaissent par rapport aux cristaux obtenus par TSSG, liés aux plus basses températures de croissance, en particulier une meilleure tenue au flux (voir http://www.crystalassociates.com/). 


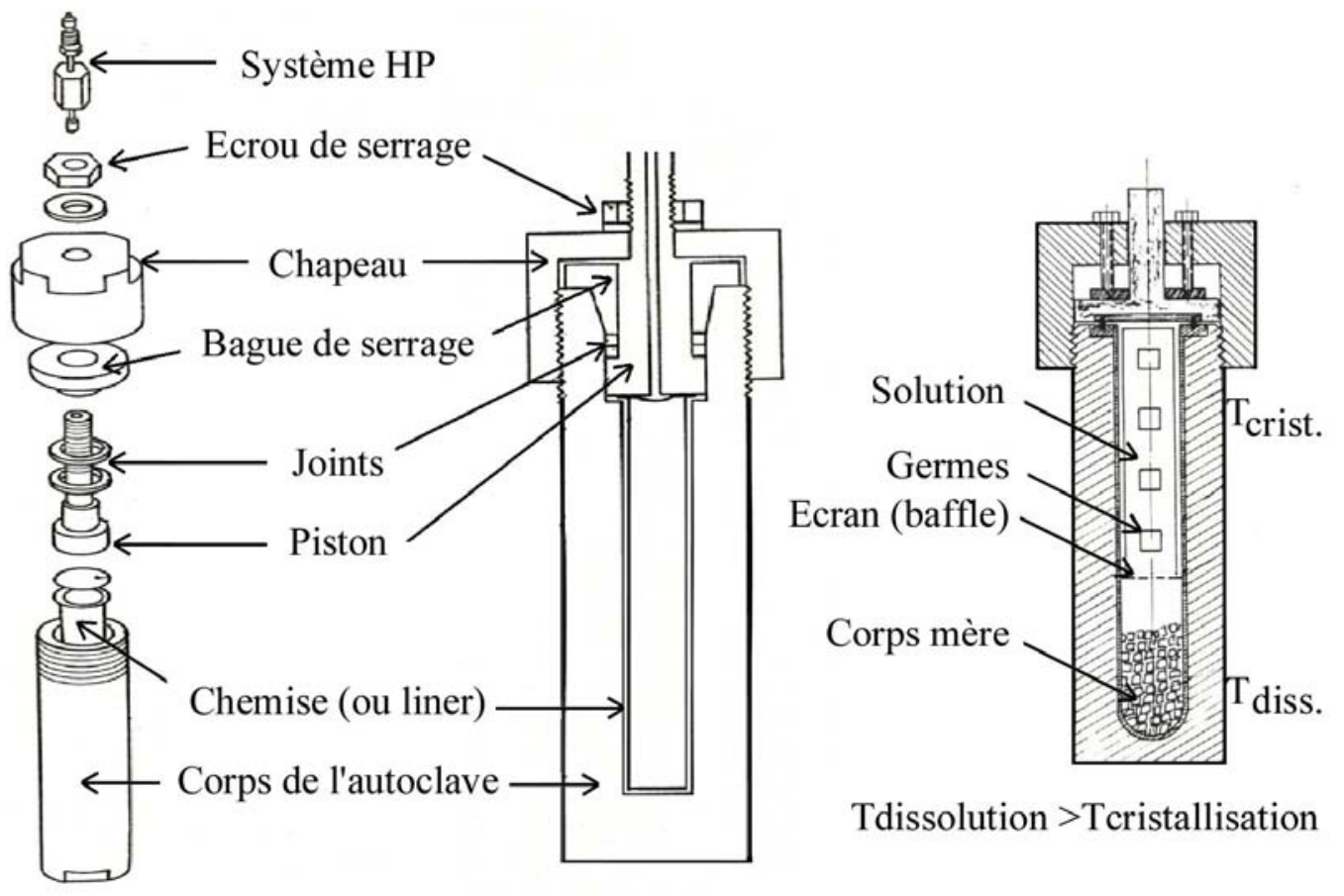

FIG. 13. Principe de la croissance hydrothermale de monocristaux.

\subsubsection{Les couches minces monocristallines}

\subsubsection{Généralités}

Depuis quelques années, les recherches concernant le développement de composants optiques miniaturisés, notamment dans les fibres et les guides d'onde planaires, s'accentuent. Les couches minces monocristallines font également l'objet de nombreuses recherches à cause des avantages qu'elles apportent : niveau de dopage plus important, gain possible plus élevé, faibles pertes... Plusieurs techniques permettent de réaliser des couches minces en modifiant localement les propriétés d'un monocristal. Ce sont la diffusion d'ions métalliques et l'échange protonique comme dans $\mathrm{LiNbO}_{3}$, l'implantation ionique, la pulvérisation RF, le dépôt par ablation laser, l'épitaxie en phase liquide. Dans ce paragraphe, on développera plus particulièrement l'épitaxie en phase liquide (ou EPL), méthode qui se rapproche beaucoup des techniques de flux présentées précédemment. On évoquera également les problèmes posés par l'adhérence moléculaire, technique très en vogue aujourd'hui pour la réalisation de nombreux dispositifs optiques.

\subsubsection{L'épitaxie en phase liquide (EPL)}

L'épitaxie en phase liquide consiste à faire croître une couche monocristalline isostructurale sur un substrat monocristallin orienté utilisé comme germe en le trempant dans une solution liquide sursaturée à température constante. Si les paramètres de maille et les coefficients de dilatation thermique de la couche et du substrat sont proches, on obtient des couches monocristallines de bonne qualité, en particulier pour les applications optiques. Plus généralement, on classe l'épitaxie en phase liquide dans les techniques de croissance en solution. Le matériau à déposer, présent sous la forme du soluté, est dissout dans un solvant. Dans la plupart des couples soluté-solvant, toute diminution de la température d'une solution initialement à l'équilibre thermodynamique doit s'accompagner de la cristallisation d'une partie du soluté pour retrouver l'équilibre. Cependant, dans de nombreux cas, la cristallisation spontanée du soluté n'intervient pas même lorsque la solution est en dessous de sa température d'équilibre dite température de saturation. Ceci s'explique par le fait que la cristallisation d'une solution passe par l'élaboration d'un germe. Mais, en dessous d'une taille critique, le système tendant à minimiser son énergie fera disparaître ce germe puisque sa croissance demanderait une augmentation de l'énergie du système. On se trouve donc dans un état «sursaturé » qui est un état métastable où la cristallisation ne peut s'installer que dans le cas d'une perturbation constituée par 
l'introduction d'un germe suffisamment gros dans la solution. C'est à cette seule condition que l'on peut initier la cristallisation et amener le système de cet état métastable vers un nouvel état d'équilibre stable.

Cette technique s'est fortement développée autour des matériaux semi-conducteurs III/V et II/VI. Les grenats sont également des matériaux à avoir été très étudiés en particulier à cause de la disponibilité de substrats de grandes dimensions et de bonne qualité cristalline (YAG, GGG...). Les solvants utilisés sont généralement un mélange de $\mathrm{PbO}$ et $\mathrm{B}_{2} \mathrm{O}_{3}$, voire $\mathrm{Bi}_{2} \mathrm{O}_{3}$. L'épitaxie en phase liquide permet de réaliser des couches diversement substituées et avec des concentrations plus élevées qu'avec les techniques de croissance des cristaux massifs [28]. Avec de telles couches monocristallines, il est possible de réaliser des guides d'onde, intéressants comme émetteurs laser ou amplificateurs. En effet leur principal intérêt est les faibles pertes obtenues $\left(0,13 \mathrm{~dB} \cdot \mathrm{cm}^{-1}\right.$ pour un guide d'onde en $\mathrm{Nd}: \mathrm{YAG})$. Outre les grenats, d'autres matériaux optiques $\left(\mathrm{LiNbO}_{3}\right.$, silicates mais également fluorures [29]) ont été préparés sous forme de couches minces monocristallines par épitaxie en phase liquide [잉.

\subsubsection{L'adhérence moléculaire}

Cette technique permet d'assembler suffisamment fortement deux matériaux très différents pour pouvoir ensuite les conditionner à volonté. Pour réaliser une telle adhésion, les deux matériaux à assembler sont polis avec une qualité «optique» et un soin particulier est apporté au nettoyage des surfaces à adhérer qui sont mises en contact. Le scellement est assuré par un traitement thermique qui vise à évacuer les gaz et autres groupements hydroxyles absorbés durant l'étape d'adhésion. Les forces de Van der Waals inter-moléculaires responsables de l'attraction des surfaces s'en trouvent ainsi renforcées.

On peut donc amincir, par la suite, deux plaques adhérées pour réaliser un guide d'onde optique. Cette technique répond à un problème important dans les applications lasers ou amplificateurs guides d'onde. Il s'agit, en effet, de faire des guides ayant une grande ouverture numérique. Ainsi, il est possible d'adhérer du Nd:YAG sur du verre ou du saphir par exemple et obtenir des ouvertures numériques de l'ordre de 0,8 qui permettent le couplage direct de diodes de puissance par exemple. Les pertes reportées dans de telles guides sont de l'ordre de $0,7 \mathrm{~dB} . \mathrm{cm}^{-1}$ pour l'assemblage Nd:YAG sur YAG ou Nd:YAG sur verre et $0,4 \mathrm{~dB} \cdot \mathrm{cm}^{-1}$ pour les assemblages Nd:GGG sur YAG [ㅍ]]

\subsection{La transformation vapeur-solide}

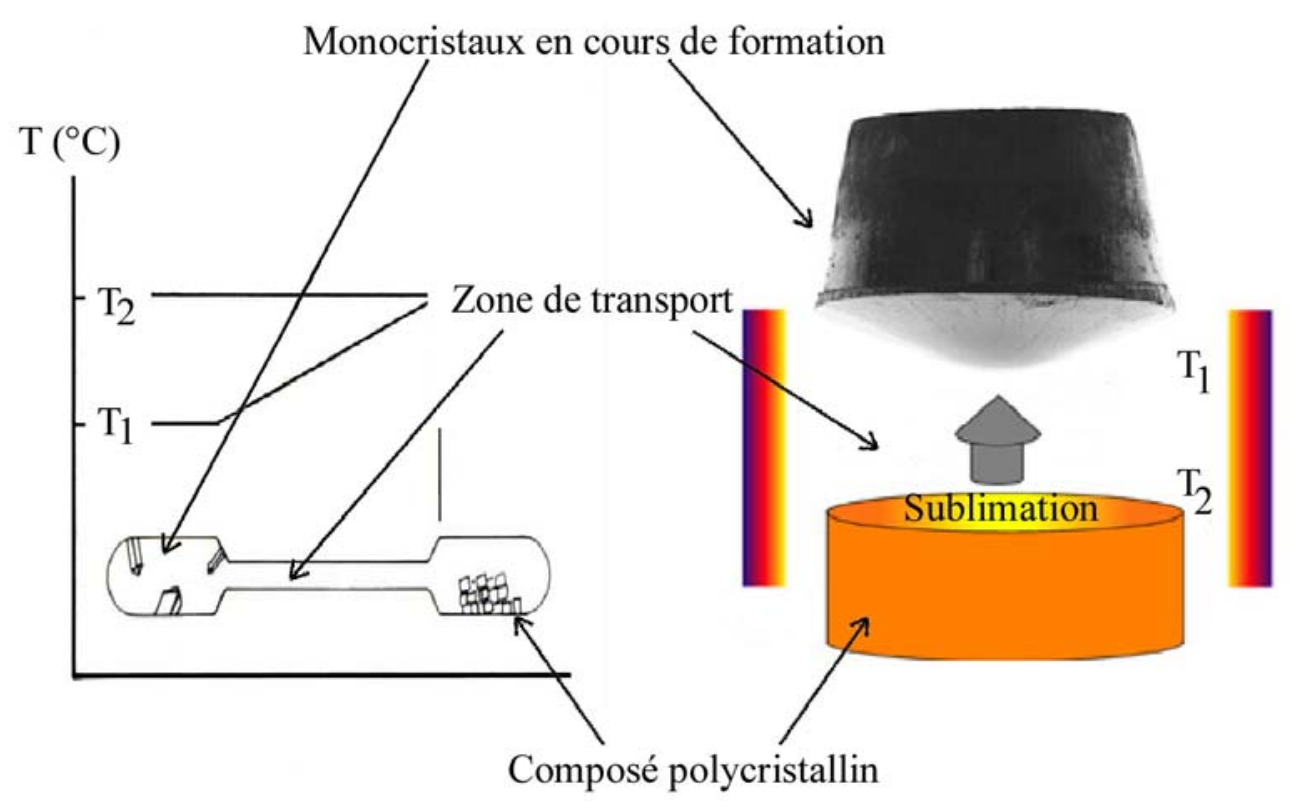

a) Évaporation

b) Sublimation

FIG. 14: Principe de la cristallisation en phase vapeur : (a) évaporation ; (b) sublimation. 
Deux méthodes principales sont utilisées pour la croissance de monocristaux en phase vapeur. On distingue la cristallisation par réaction en phase gazeuse et la croissance par condensation d'un matériau évaporé ou sublimé (Fig. 14). La première méthode, utilisée pour la préparation de couches minces et la purification, n'a connu qu'un développement restreint en raison de la petite taille des cristaux obtenus. $\mathrm{HgI}_{2}$ a été un cristal cas d'école pour cette méthode. La deuxième méthode est relativement utilisée pour la croissance de sulfures ou séléniures. Aujourd'hui la croissance du carbure de silicium $(\mathrm{SiC})$ a fortement relancé la technique de sublimation, appelée Lely modifiée [32]. Elle permet dans un creuset en graphite purifié d'obtenir une croissance contrôlée sur germe de monocristaux des différents polytypes : $\mathrm{SiC} 6 \mathrm{H}$ et $4 \mathrm{H}$. La société américaine $\mathrm{CREE}$ est leader mondial dans le domaine. Des monocristaux de dimensions $50 \mathrm{~mm}$ et plus, ayant un faible taux de dislocation $\left(<1000 / \mathrm{cm}^{2}\right)$ et une densité de micropipes inférieure à $10 / \mathrm{cm}^{2}$, ont pu être ainsi obtenus et commercialisés.

\section{CONCLUSION}

Dans cet article, nous avons présenté les principales techniques de croissance cristalline en s'attachant à donner des exemples de cristaux ioniques ayant des applications en optique. La cristallogenèse reste encore un art autant qu'une science. C'est pourquoi un nombre considérable de méthodes se sont développées autour des nombreux monocristaux qui présentent un intérêt potentiel en optique. Nous avons discuté des méthodes les plus connues faisant appel principalement à une transformation liquide-solide. Parmi elles, on peut distinguer les méthodes à croissance rapide et les méthodes à croissance lente. Les méthodes à croissance rapide (quelques $\mathrm{mm} / \mathrm{h}$ ) opèrent aux températures élevées permettant d'obtenir de gros monocristaux et sont généralement caractérisées par une croissance dirigée à partir d'un bain fondu. Les méthodes à croissance lente (quelques $\mathrm{mm} / \mathrm{jour}$ ) fonctionnent à des températures plus basses et s'imposent quand la fusion du cristal n'est pas congruente ou lorsque le matériau présente une transition de phase à haute température. Ce sont généralement les méthodes de croissance en solution faisant appel à un solvant et la synthèse hydrothermale. Indépendamment des considérations de fusion et de transition de phase, nous avons vu que chaque matériau nécessite des conditions de croissance particulières qui peuvent influer fortement sur leur coût et en conséquence sur celui des dispositifs.

Dans un article général qui parle de monocristaux, on ne peut pas passer sous silence les problèmes liés à leur mise en forme pour leur utilisation (Fig. 15). En effet de nombreuses applications optiques font appel à des monocristaux ayant des formes très particulières (barreaux, prismes, lames à faces parallèles...) et des états de surface très soignés (rugosité, propreté...). Les dispositifs d'usinage et de polissage utilisés pour le conditionnement des monocristaux sont généralement associés à un diffractomètre à rayons- $\mathrm{X}$ car certaines applications (doublage de fréquences par exemple) nécessitent de plus des orientations cristallographiques très précises.

Les applications des monocristaux en optique sont très nombreuses: émetteurs laser, convertisseurs de fréquences, modulateurs électro-optiques et acousto-optiques, composants photoréfractifs, rotateurs magnéto-optiques, scintillateurs, polariseurs, prismes, lentilles, miroirs, fenêtres ou substrats. Bien que de nombreux monocristaux répondent généralement aux critères de choix de ces différentes applications, seuls quelques-uns $\left(\mathrm{Y}_{3} \mathrm{Al}_{5} \mathrm{O}_{12}, \mathrm{LiNbO}_{3}, \mathrm{Al}_{2} \mathrm{O}_{3}, \ldots\right)$ ont un développement industriel important, car c'est bien souvent la réussite ou non de la cristallogenèse (dimensions, qualité) qui décide de l'avenir industriel d'un matériau. En effet bien que faisant beaucoup de progrès, notamment dans le domaine de la compréhension des phénomènes de croissance et de l'amélioration des procédés, la cristallogenèse n'est pas toujours aisée à mettre en place et reste encore un art dont la science n'a pas encore élucidé tous les mystères. 


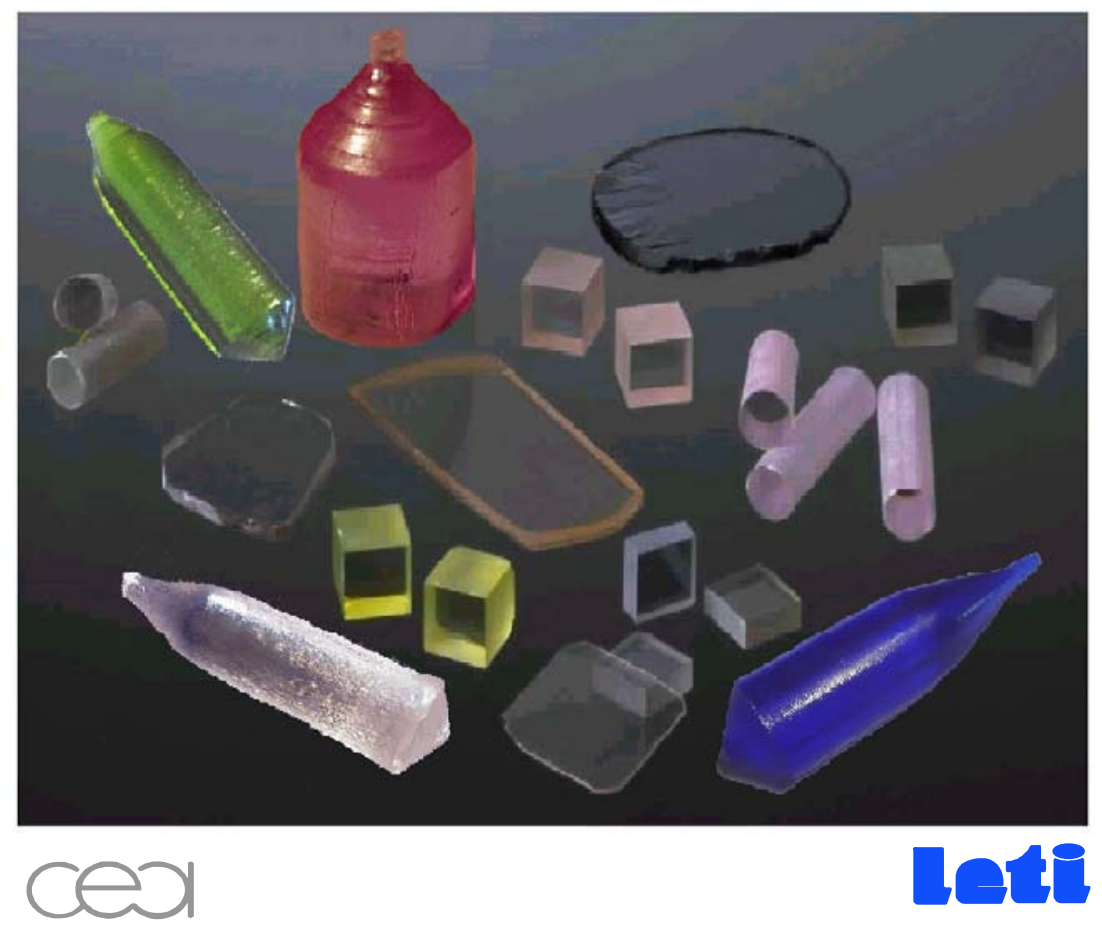

FIG. 15 : Monocristaux d'oxydes bruts de croissance et conditionnés pour diverses applications.

\section{REMERCIEMENTS}

Je voudrais remercier tous les collègues des différents laboratoires de cristallogenèse français qui m'ont permis de réaliser ce tour d'horizon (sans doute pas complet) des différentes méthodes de fabrication de monocristaux utilisés en optique : Daniel Vivien et Gérard Aka à l'ENSC Paris, Georges Boulon et Marie-Thérèse Cohen Addad à l'UCB Lyon, Richard Moncorgé à l'Université de Caen, Jean-Pierre Chaminade à l'ICMC Bordeaux, Thierry Duffar à l'INP Grenoble, Jean-Louis Santailler au CEA-Grenoble. Un certain nombre de détails donnés dans ce cours ont été empruntés aux travaux réalisés dans ces différents laboratoires.

Je ne peux pas terminer cet article sans mentionner tous ceux avec qui j'ai travaillé tout au long de ces années : Jean-Claude Joubert, Jean Mareschal et Jacques Daval pour tout ce qu'ils m'ont appris en cristallogenèse, Jean-Jacques Aubert, Christophe Wyon, Denis Pelenc, Yves Grange, Maurice Couchaud, Claude Calvat, Gérard Basset, Christian Faure, Bernard Chambaz, Alain Basset et Philippe Hugot pour tous les monocristaux que nous avons fabriqués ensemble.

\section{RÉFÉRENCES}

[1] A. Verneuil, Annales de Chimie et de Physique $\mathbf{8}^{\mathrm{e}}$ série III (1904), 20.

[2] J. Czochralski, Z. Physik Chem. 92 (1918) 219.

[3] M. Spiesser, Jan Czochralski et la méthode du tirage des cristaux, Bulletin GFCC (Mai 1999).

[4] D. Elwell et H.J. Scheel, Crystal growth from high-temperature solutions, New York Academic Press (1975).

[5] J.C. Bouillard, Et l'homme créa la pierre (Collections de Minéraux), Université Pierre et Marie Curie (1996).

[6] H.J. Scheel, Historical aspects of crystal growth technology, J. Cryst. Growth 211 (2000) 1.

[7] R.A. Laudise, The growth of single crystal, Prentice Hall, Englewood Cliffs, New Jersey (1970). 
[8] G.R. Teal et J.B. Little, Growth of germanium single crystals, Phys. Rev. 78 (1950) 647.

[9] T.H. Maiman, Stimulated optical radiation in ruby, Nature 187 (1960) 493.

[10] L.F. Johnson et K. Nassau, First continuous operation of an optically pumped solid-state laser in Nd:CaWO 4 at 1.06 microns, Proc. IRE 49 (1961) 1704.

[11] D. Vivien, Les matériaux laser solides, Ann. Chim. Fr. 20 (1995) 211.

[12] Growth and characterization of non-linear optical materials, Progress in Crystal Growth and Characterization of Materials 20, 1/2 (1990) 1.

[13] R.F. Belt, R.C. Puttbach et D.A. Lepore, Crystal growth and perfection of large Czochalski Nd:YAG single crystals, J. Cryst. Growth 13/14 (1972) 268.

[14] C.D. Brandle et D. C. Miller, Czochralski growth of large diameter $\mathrm{LiTaO}_{3}$ crystals, J. Cryst. Growth 24/25 (1974) 432.

[15] B. Cockayne, J.G. Plant et R.A. Clay, The Czochralski growth and laser characteristics of LiYF 4 scheelite single crystals, J. Cryst. Growth 54 (1981) 407.

[16] J.B. Mullin, B.W. Straughan et W.S. Brickell, Liquid Encapsulation Techniques: The use of an inert liquid in suppressing dissociation during the melt growth of InAs, GaAs crystals, J. Phys. Chem. Solids 26 (1965) 782.

[17] C.P. Khattak et F. Schmid, Growth of large diameter crystals by HEM for optical and laser applications, SPIE 505, Advances in Optical Materials (1984) 4.

[18] A. Ocafrain, Mise en oeuvre et utilisation de la méthode de l'échangeur thermique (HEM) pour l'obtention de monocristaux à applications spécifiques et de céramiques supraconductrices orientées, Thèse, Université Bordeaux, France (1997).

[19] R.S. Feigelson, Pulling optical fibers, J. Cryst. Growth 79 (1986) 669.

[20] G. Foulon, Fibres monocristallines lasers de niobates à susceptibilités diélectriques non linéaires $\chi^{(2)}$ élevées, purs ou dopés par des ions terres rares, Thèse, Université Claude Bernard Lyon I, France (1996).

[21] V.A. Tartachenko, Capillary shaping in crystal growth from melts, J. Cryst. Growth 37 (1977) 272.

[22] H.E. LaBelle, EFG: the invention and application to sapphire growth, J. Cryst. Growth $\mathbf{5 0}$ (1980) 8.

[23] F. Théodore, Préformage de cristaux de saphir optique, Thèse, INP Grenoble, France (1998).

[24] National Ignition Facility Project. Livermore CA, http://www.llnl.gov/nif/nif.html.

[25] W. Tolksdorf, Growth of magnetic garnet single crystals from high temperature solution, J. Cryst. Growth 42 (1977) 275.

[26] D. Elwell, P. Capper et M. D’Agostino, Apparatus for Top-Seeded Growth from high temperature solutions, J. Cryst. Growth 29 (1975) 263.

[27] R.A. Laudise et J.W. Nielsen, Hydrothermal crystal growth, Solid State Physics 12 (1961) 149.

[28] B. Ferrand, D. Pelenc, I. Chartier et C. Wyon, Growth by LPE of Nd:YAG single crystal layers for waveguide laser applications, J. Cryst. Growth 128 (1993) 966.

[29] R. Burkhalter, I. Dohnke et J. Hulliger, Growing of bulk crystals and structuring waveguides of fluoride materials for laser application, Progress in Crystal Growth and Characterization of Materials 42, 1/2 (2001) 1 .

[30] B. Ferrand, B. Chambaz et M. Couchaud, Liquid Phase Epitaxy: a versatile technique for the development of miniature optical components in single crystal dielectric media, Optical Materials 11 (1999) 101. 
[31] D.P. Shepherd, C.L. Bonner, C.T.A. Brown, W.A. Clarkson, A.C. Tropper, D.C. Hanna et H.E. Meissner, High-numerical-aperture, contact-bonded, planar waveguides for diode-bar-pumped lasers, Opt. Commun. 160 (1999) 47.

[32] Y.M. Tairov et V.F. Tsvetkov, Investigation of growth process of ingots of silicon carbide single crystals, J. Cryst. Growth 43 (1978) 209. 